\title{
Quantitative Analysis of HIV-1 Protease Inhibitors in Cell Lysates Using MALDI-FTICR Mass Spectrometry
}

\author{
Jeroen J. A. van Kampen, ${ }^{\dagger, \neq}$ Peter C. Burgers, ${ }^{\dagger}$ Ronald de Groot, ${ }^{\S}$ Albert D. M. E. Osterhaus," \\ Mariska L. Reedijk," Esther J. Verschuren," Rob A. Gruters," and Theo M. Luider*,t

\begin{abstract}
Department of Neurology, Laboratory of Neuro-Oncology and Clinical and Cancer Proteomics and Department of Virology, Erasmus MC, Rotterdam, The Netherlands, Department of Pediatrics, Division of Pediatric Infectious Diseases \& Immunology, Erasmus MC-Sophia, Rotterdam, The Netherlands, and Department of Pediatrics, UMC St. Radboud, Nijmegen, The Netherlands
\end{abstract}

In this report we explore the use of MALDI-FTICR mass spectrometry for the quantitative analysis of five HIV-1 protease inhibitors in cell lysates. 2,5-Dihydroxybenzoic acid (DHB) was used as the matrix. From a quantitative perspective, DHB is usually a poor matrix due to its poor shot-to-shot and poor spot-to-spot reproducibilities. We found that the quantitative precisions improved significantly when DMSO (dimethylsulfoxide) was added to the matrix solution. For lopinavir and ritonavir, currently the most frequently prescribed HIV-1 protease inhibitors, the signal-to-noise ratios improved significantly when potassium iodide was added to the matrix solution. The mean quantitative precisions, expressed as \% relative standard deviation, were $6.4 \%$ for saquinavir, $7.3 \%$ for lopinavir, $\mathbf{8 . 5} \%$ for ritonavir, $11.1 \%$ for indinavir, and $7.2 \%$ for nelfinavir. The mean quantitative accuracies, expressed as \% deviation, were $4.5 \%$ for saquinavir, $6.0 \%$ for lopinavir, $5.9 \%$ for ritonavir, $6.6 \%$ for indinavir, and $8.0 \%$ for nelfinavir. The concentrations measured for the individual quality control samples were all within $85-117 \%$ of the theoretical concentrations. The lower limits of quantification in cell lysates were $4 \mathrm{fmol} / \mu \mathrm{L}$ for saquinavir, $16 \mathrm{fmol} / \mu \mathrm{L}$ for lopinavir, $31 \mathrm{fmol} / \mu \mathrm{L}$ for ritonavir, and $100 \mathrm{fmol} / \mu \mathrm{L}$ for indinavir and nelfinavir. The mean mass accuracies for the protease inhibitors were $\leqslant 0.28 \mathrm{ppm}$ using external calibration. Our results show that MALDIFTICR mass spectrometry can be successfully used for precise, accurate, and selective quantitative analyses of HIV-1 protease inhibitors in cell lysates. In addition, the lower limits of quantification obtained allow clinical applications of the technique.

Quantitative drug analysis using matrix-assisted laser desorption/ionization (MALDI) mass spectrometry is normally hampered

\footnotetext{
* Corresponding author. Theo M. Luider, PhD, Room BE 462, Erasmus MC, P.O. Box 1738, 3000 DR Rotterdam. Phone: +31107038069 . Fax: +31 107038365 E-mail: t.luider@erasmusmc.nl. MC.

Laboratory of Neuro-Oncology and Clinical and Cancer Proteomics, Erasmus

* Erasmus MC-Sophia.

$\$$ UMC St. Radboud.

"Department of Virology, Erasmus MC.
}

by poor reproducibility of the signal intensities and by the presence of matrix-derived signals (leading to the so-called chemical noise) in the low mass range, i.e., below $1000 \mathrm{Da}$. As a consequence, electrospray ionization (ESI), in particular in combination with a triple quadrupole mass analyzer, is normally preferred. When operated in the selected reaction monitoring mode (SRM), the triple quadrupole mass analyzer selectively measures unique fragments of the analyte(s) and internal standard(s). The ESI ion source can be coupled to a high pressure liquid chromatograph (HPLC), which further increases the assay's selectivity with concomitant decrease of ion suppression effects.

However, MALDI offers certain advantages over ESI for the quantitative analysis of drugs as well as for the analysis of other compounds: it is capable of a higher sample throughput, ${ }^{1}$ samples can be conveniently stored on the target plate for future reanalysis, ${ }^{2}$ and MALDI is less susceptible to ion suppression. ${ }^{3}$

Various approaches have been developed to improve the performance of MALDI for the quantitative analysis of drugs, which aim to eliminate the disadvantages normally associated with MALDI, viz., the poor reproducibility of signal intensities and the presence of matrix-derived chemical noise. ${ }^{4}$ Thus, high molecular weight matrixes, ${ }^{5-9}$ additives, ${ }^{10}$ and matrixless target plates ${ }^{11,12}$ have been used to decrease and even to eliminate the matrixderived chemical noise in the low mass range. The reproducibility

(1) Volmer, D. A.; Sleno, L.; Bateman, K.; Sturino, C.; Oballa, R.; Mauriala, T.; Corr, J. Anal. Chem. 2007, 79, 9000-9006.

(2) Dekker, L. J.; Burgers, P. C.; Guzel, C.; Luider, T. M. L. Chromatogr., B: Anal. Technol. Biomed. Life Sci. 2007, 847, 62-64.

(3) Siuzdak, G. Mass Spectrometry for Biotechnology, 1st ed.; Academic Press: San Diego, CA, 1996.

(4) Cohen, L. H.; Gusev, A. I. Anal. Bioanal. Chem. 2002, 373, 571-586.

(5) Chen, Y. T.; Ling, Y. C. L. Mass Spectrom. 2002, 37, 716-730.

(6) Ling, Y. C.; Lin, L.; Chen, Y. T. Rapid Commun. Mass Spectrom. 1998, 12, 317-327.

(7) Ayorinde, F. O.; Hambright, P.; Porter, T. N.; Keith, Q. L., Jr. Rapid Commun. Mass Spectrom. 1999, 13, 2474-2479.

(8) van Kampen, J. J.; Burgers, P. C.; de Groot, R.; Luider, T. M. Anal. Chem. 2006, 78, 5403-5411.

(9) van Kampen, J. J.; Verschuren, E. J.; Burgers, P. C.; Luider, T. M.; de Groot, R.; Osterhaus, A. D.; Gruters, R. A. I. Chromatogr., B: Anal. Technol. Biomed. Life Sci. 2007, 847, 38-44.

(10) Guo, Z.; Zhang, Q.; Zou, H.; Guo, B.; Ni, J. Anal. Chem. 2002, 74, 16371641.

(11) Wei, J.; Buriak, J. M.; Siuzdak, G. Nature 1999, 399, 243-246.

(12) Kang, M. J.; Pyun, J. C.; Lee, J. C.; Choi, Y. J.; Park, J. H.; Park, J. G.; Lee, J. G.; Choi, H. J. Rapid Commun. Mass Spectrom. 2005, 19, 3166-3170. 
can be significantly increased by using ionic liquid matrixes, ${ }^{13}$ internal standards, sophisticated sample/matrix spotting devices ${ }^{14}$ and prestructured target plates, ${ }^{15}$ or by averaging out many spectra of a single sample. These approaches have led to the successful development of various quantitative drug assays using MALDI-TOF, ${ }^{8,9,16,17}$ MALDI-q-TOF, ${ }^{18,19}$ and MALDI-triple quadrupole ${ }^{1,18,20,21}$ mass spectrometry.

Currently, no assays have been described which use MALDIFTICR for the quantitative analysis of drugs. The advantage of MALDI-FTICR over other types of MALDI mass spectrometers results from its high resolving power and mass accuracy, which significantly increase the selectivity of the assay; in particular this will be the case when the molecular ions of the analyte and internal standard are used for quantitative analysis, i.e., from a full mass spectrum (MS mode).

In this study, we assess the quantitative performance of MALDI-FTICR mass spectrometry for the analysis of HIV-1 protease inhibitors in lysates of peripheral blood mononuclear cells (PBMCs).

\section{EXPERIMENTAL SECTION}

Chemicals. Lopinavir (LPV; $\mathrm{C}_{37} \mathrm{H}_{48} \mathrm{~N}_{4} \mathrm{O}_{5}$; monoisotopic molecular mass $628.36247 \mathrm{Da}$ ) and ritonavir (RTV; $\mathrm{C}_{37} \mathrm{H}_{48} \mathrm{~N}_{6} \mathrm{O}_{5} \mathrm{~S}_{2}$; monoisotopic molecular mass $720.31276 \mathrm{Da}$ ) were kindly donated by Abbott Laboratories (Illinois). Saquinavir (SQV; $\mathrm{C}_{38} \mathrm{H}_{50} \mathrm{~N}_{6} \mathrm{O}_{5}$; monoisotopic molecular mass $670.38427 \mathrm{Da}$ ) was kindly donated by F. Hoffmann-La Roche (Basel, Switzerland). Nelfinavir (NFV; $\mathrm{C}_{32} \mathrm{H}_{45} \mathrm{~N}_{3} \mathrm{O}_{4} \mathrm{~S}$; monoisotopic molecular mass $567.31308 \mathrm{Da}$ ) was kindly donated by Pfizer (Groton, CT), and indinavir (IDV; $\mathrm{C}_{36} \mathrm{H}_{47} \mathrm{~N}_{5} \mathrm{O}_{4}$; monoisotopic molecular mass $613.36281 \mathrm{Da}$ ) was kindly donated by Merck (Rahway, NJ). Potassium iodide was obtained from Sigma-Aldrich. 2,5-Dihydroxybenzoic acid (DHB) was obtained from Bruker Daltonics (Germany).

Peripheral Blood Mononuclear Cells. Peripheral blood mononuclear cells (PBMCs) were obtained from a buffy coat (Sanquin, Rotterdam, The Netherlands) using a standard Ficoll density gradient. The PBMCs were extracted overnight in methanol at $5{ }^{\circ} \mathrm{C}$. The next day, the PBMC lysates were collected, water was added to the samples until the water-to-methanol ratio was 3:1, and the PBMC lysates were loaded onto a 96-well solid phase extraction plate (Oasis HLB $\mu$ elution plate, Waters). Subsequently, the samples were washed twice with $200 \mu \mathrm{L}$ of methanol/water $(1: 3 \mathrm{v} / \mathrm{v})$. Next, the samples were eluted from the column with $100 \mu \mathrm{L}$ of methanol. The samples were dried in a SpeedVac (Savant) and stored at $-80^{\circ} \mathrm{C}$ until the day of analysis.

(13) Tholey, A.; Heinzle, E. Anal. Bioanal. Chem. 2006, 386, 24-37.

(14) Hensel, R. R.; King, R. C.; Owens, K. G. Rapid Commun. Mass Spectrom. 1997, 11, 1785-1793.

(15) Schuerenberg, M.; Luebbert, C.; Eickhoff, H.; Kalkum, M.; Lehrach, H.; Nordhoff, E. Anal. Chem. 2000, 72, 3436-3442.

(16) Duncan, M. W.; Matanovic, G.; Cerpa-Poljak, A. Rapid Commun. Mass Spectrom. 1993, 7, 1090-1094.

(17) Rideout, D.; Bustamante, A.; Siuzdak, G. Proc. Natl. Acad. Sci. U.S.A. 1993, 90, 10226-10229.

(18) Hatsis, P.; Brombacher, S.; Corr, J.; Kovarik, P.; Volmer, D. A. $\underline{\text { Rapid }}$ Commun. Mass Spectrom. 2003, 17, 2303-2309.

(19) Notari, S.; Mancone, C.; Tripodi, M.; Narciso, P.; Fasano, M.; Ascenzi, P. L. Chromatogr., B: Anal. Technol. Biomed. Life Sci. 2006, 833, 109-116.

(20) Gobey, J.; Cole, M.; Janiszewski, J.; Covey, T.; Chau, T.; Kovarik, P.; Corr, J. Anal. Chem. 2005, 77, 5643-5654.

(21) Sleno, L; Volmer, D. A. Anal. Chem. 2005, 77, 1509-1517.
Mass Spectrometry. To study the effect of various DHB preparations on the signal intensities and \% $\mathrm{CV}$ of the analyte-tointernal standard ratios, a mixture of five pure HIV protease inhibitors was analyzed with four different DHB preparations: $\mathrm{DHB}=10 \mathrm{mg} / \mathrm{mL}$ DHB in MeCN/water (1/1); DHB + DMSO $=10 \mathrm{mg} / \mathrm{mL} \mathrm{DHB}$ in MeCN$/$ water/DMSO (9/9/2); DHB + KI $=10 \mathrm{mg} / \mathrm{mL} \mathrm{DHB}+10 \mathrm{mM} \mathrm{KI}$ in MeCN/water (1/1); DHB + $\mathrm{KI}+\mathrm{DMSO}=10 \mathrm{mg} / \mathrm{mL} \mathrm{DHB}+10 \mathrm{mM} \mathrm{KI}$ in $\mathrm{MeCN} /$ water/ DMSO (9/9/2). For each DHB preparation, four technical replicates, i.e., four spots on the target plate, were measured (1 $\mu \mathrm{L}$ per spot).

For quantitative analysis of HIV protease inhibitors in cell lysates, the samples were prepared by reconstituting the dried PBMC lysates in $10 \mu \mathrm{L}$ of water/MeCN/DMSO (9/9/2 v/v/v) containing $10 \mathrm{mg} / \mathrm{mL} \mathrm{DHB}, 10 \mathrm{mM} \mathrm{KI}$, analyte at various concentrations, and $1 \mathrm{pmol} / \mu \mathrm{L}$ internal standard. Technical replicates of each calibrator were spotted on different positions on a $400 \mu \mathrm{m}$ AnchorChip target plate (Bruker Daltonics, Germany) $(1 \mu \mathrm{L}$ per spot). Drying of the samples was assisted with a heat blower.

Samples were measured on an APEX IV Qe 9.4 T FTICR mass spectrometer (Bruker Daltonics) equipped with the first version of the vacuum Combisource and a $20 \mathrm{~Hz}$ nitrogen laser with irradiation area of $\sim 200 \mu \mathrm{m} .{ }^{22}$ Xmass version 7.0.8 was used to operate the mass spectrometer, and DataAnalysis version 3.4 was used for data analysis (both from Bruker Daltonics). A multishot accumulation was used as recommended by O'Connor et al., Mize et al., and Moyer et al. ${ }^{23-25}$ Ions produced by 10 laser shots were accumulated in the storage hexapole, transferred to the FTICR cell, and scanned for $0.78 \mathrm{~s}$ (TD size $512 \mathrm{~Kb}$ ). Fifty scans were summed for each mass spectrum. The acquisition mass range was $m / z 500-1000$. The mass spectra were subsequently apodized and zerofilled twice. An external mass calibration was applied using a quadratic equation.

Quantitative Analysis of HIV Protease Inhibitors in Cell Lysates. Two data sets (A and B) were used to assess the quantitative performance of the MALDI-FTICR assay. Data set A was used to construct the calibration curve while data set B was used as quality controls and vice versa. For each sample in a data set, three spots on the target plate, i.e., three technical replicates, were measured. The quantitative precisions were obtained by calculating the \% relative standard deviation of the analyte-tointernal standard ratio of the three replicate analyses of each sample. The mean analyte-to-internal standard ratios of the three replicate analyses were used to construct the calibration curve (calibrators) or to check the quantitative accuracies (quality controls). The mean analyte-to-internal standard ratios and analyte concentrations were log 10-transformed, and a linear unweighed curve was fitted through the data points.

\section{RESULTS AND DISCUSSION}

Choice of Matrix. Small molecule analysis by MALDI mass spectrometry is normally hampered by the presence of matrix-

(22) Baykut, G.; Jertz, R.; Witt, M. Rapid Commun. Mass Spectrom. 2000, 14, 1238-1247.

(23) Mize, T. H.; Amster, I. J. Anal. Chem. 2000, 72, 5886-5891.

(24) Moyer, S. C.; Budnik, B. A.; Pittman, J. L.; Costello, C. E.; O’Connor, P. B. Anal. Chem. 2003, 75, 6449-6454.

(25) O'Connor, P. B.; Costello, C. E. Anal. Chem. 2000, 72, 5125-5130. 


\begin{tabular}{|c|c|c|c|c|c|}
\hline & $\begin{array}{c}\text { amount } \\
\text { (femtomole) }\end{array}$ & $\begin{array}{c}\text { DHB } \\
{\text { [analyte }+\mathrm{H}^{+}}^{+}\end{array}$ & 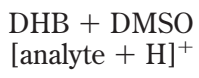 & $\begin{array}{c}\mathrm{DHB}+\mathrm{KI} \\
{[\text { analyte }+\mathrm{K}]^{+}}\end{array}$ & $\begin{array}{l}\mathrm{DHB}+\mathrm{KI}+\mathrm{DMSO} \\
\quad \text { analyte }^{-} \mathrm{K}^{+}\end{array}$ \\
\hline nelfinavir & 1000 & 5179 & 2151 & 552 & 745 \\
\hline indinavir & 1000 & 1363 & 1820 & 212 & 627 \\
\hline lopinavir & 1000 & 284 & 149 & 1680 & 2283 \\
\hline saquinavir & 250 & 5476 & 5924 & 1125 & 1597 \\
\hline ritonavir & 1000 & 479 & 632 & 845 & 2933 \\
\hline
\end{tabular}

${ }^{a}$ A mixture of five pure HIV-1 protease inhibitors was analyzed using four different DHB preparations (see Experimental Section). The concentration per spot on the target plate was 1 pmol for nelfinavir, indinavir, lopinavir, and ritonavir and 250 fmol for saquinavir. Four technical replicates, i.e., four different spots on the target plate, were measured for each sample.

derived chemical noise in the low mass range, i.e., below 1000 $\mathrm{Da}^{26}$ the bane of any MALDI practitioner. The use of a high molecular weight matrix is one way to overcome this phenomenon. ${ }^{7}$ We have recently developed a quantitative assay for HIV protease inhibitors on a MALDI-TOF mass spectrometer using the high molecular weight matrix meso-tetrakis(pentafluorophenyl)porphyrin (F20TPP) ${ }^{8,9}$ The molecular weight of F20TPP is 974.6 Da, and so the only matrix-derived peaks which can be observed in the low mass range result from dissociation of the matrix or from matrix impurities. Thus, using a TOF analyzer, we observed, as expected, only a few peaks in the low mass range, making F20TPP eminently suitable as a matrix in TOF analyses. We also attempted to use the F20TPP matrix on our MALDIFTICR mass spectrometer. In contrast to our previous MALDITOF experiments, extensive fragmentation of the F20TPP matrix was observed in our FTICR experiments which resulted in matrixderived interfering signals in the low mass range. Long-lived metastable decay of ions formed in the MALDI source has been extensively reported in the literature. ${ }^{27-34}$ The metastable fragmentations in FTICR are the result of the relatively long ion lifetime ( $1 \mathrm{~s})$ of the ions prior to detection in FTICR, compared to the much shorter lifetimes associated with more conventional MS techniques, such as TOF $\left(10^{-4} \mathrm{~s}\right)$, and thus they are a direct consequence of RRKM theory. ${ }^{35}$ For example, ions with a rate constant $(k)$ of $10^{3} \mathrm{~s}^{-1}$ fragment on average in $10^{-3}$ seconds. ${ }^{36}$ These ions formed in the MALDI process could thus easily survive the TOF time frame but would generate extensive fragmentation in FTICR. ${ }^{28}$ In the same vein, noncovalent matrix adduct ions are expected to be metastable on the FTICR time frame, and dissociation is thus expected. This indeed appears to be the case; using our FTICR instrument, the matrix DHB hardly shows any

(26) Krutchinsky, A. N.; Chait, B. T. I.Am. Soc. Mass Spectrom. 2002, 13, 129134.

(27) Stemmler, E. A.; Buchanan, M. V.; Hurst, G. B.; Hettich, R. L. Anal. Chem. 1995, 67, 2924-2930.

(28) O’Connor, P. B.; Costello, C. E Rapid Commun. Mass Spectrom. 2001, 15, $1862-1868$.

(29) Ho, Y. P.; Fenselau, C. I. Mass Spectrom. 2000, 35, 183-188.

(30) Penn, S. G.; Cancilla, M. T.; Lebrilla, C. B. Anal. Chem. 1996, 68, 23312339.

(31) Cancilla, M. T.; Penn, S. G.; Lebrilla, C. B. Anal. Chem. 1998, 70, 663672.

(32) Wong, A. W.; Cancilla, M. T.; Voss, L. R.; Lebrilla, C. B. Anal. Chem. 1999, $71,205-211$.

(33) Cancilla, M. T.; Wong, A. W.; Voss, L. R.; Lebrilla, C. B. Anal. Chem. 1999, 71, 3206-3218.

(34) Wong, A. W.; Wang, H.; Lebrilla, C. B. Anal. Chem. 2000, 72, 1419-1425.

(35) Rosenstock, H. M.; Wallenstein, M. B.; Wahrhaftig, A. L.; Eyring, H. Proc. Natl. Acad. Sci. U.S.A. 1952, 38, 667-678.

(36) Gross, J. H. In Mass Spectrometry: A Textbook, 1st ed.; Gross, J. H., Ed.; Springer: New York, 2004; pp 28-29. chemical noise. Therefore, DHB was tested as a matrix for the FTICR analysis of the HIV-1 protease inhibitors.

Matrix Preparation. We evaluated four different matrix solutions: DHB only, DHB with DMSO, DHB with KI, and DHB with both DMSO and KI, see Table 1. As can be seen from this table, the highest $\mathrm{S} / \mathrm{N}$ ratios were obtained for the protonated forms of nelfinavir, indinavir, and saquinavir, i.e., when DHB was used without the addition of KI. In contrast, the highest $\mathrm{S} / \mathrm{N}$ ratios for lopinavir and ritonavir were observed when DHB was used with the addition of KI. DHB crystallizes in an inhomogeneous way when it is spotted according to the widely used dried droplet protocol (at ambient temperature). Because of this inhomogeneous crystallization, one needs to search for so-called sweet spots in the sample/matrix crystals. In general, better quantitative precisions are obtained when the sample/matrix crystals have a homogeneous appearance. Therefore, we added DMSO to the matrix solution. With the use of DMSO, the samples dry at a much slower rate and dense homogeneous DHB crystals are formed, even when dried using a heat blower. As shown in Table 2, the quantitative precisions improved significantly when DMSO was added to the DHB solution. In addition, as shown in Table 1, the $\mathrm{S} / \mathrm{N}$ of the potassiated HIV-1 protease inhibitors also improved by adding DMSO to the matrix solution.

Quantitative Analysis of HIV Protease Inhibitors in Cell Lysates. Our goal was to develop one assay for the quantitative analysis of five HIV-1 protease inhibitors. As shown above, the highest $\mathrm{S} / \mathrm{N}$ values were obtained for the protonated forms of nelfinavir, indinavir, and saquinavir, while lopinavir and ritonavir were best detected as potassiated ions. Thus a tradeoff was needed in the preparation of the DHB solution, i.e., preparation with or without potassium iodide. Currently, Kaletra is the most widely used HIV-1 protease inhibitor. Kaletra is a preparation of lopinavir together with ritonavir which serves as a pharmacokinetic booster. Because of the clinical importance of lopinavir and ritonavir, we added potassium iodide to the matrix solution for the quantitative analysis of HIV-1 protease inhibitors in lysates of peripheral blood mononuclear cells. In addition, DMSO was added to the matrix solution to enhance the quantitative precisions.

Stable isotope labeled internal standards would serve as the best internal standards for quantitative MALDI experiments. However, these were not commercially available for the tested HIV-1 protease inhibitors. Therefore, we chose to use nelfinavir as the internal standard for the quantitative analysis of indinavir, lopinavir, saquinavir, and ritonavir. For the quantitative analysis of nelfinavir, we used indinavir as the internal standard. Figure 1 shows the mass spectrum of lopinavir and nelfinavir (internal 
Table 2. The Effect of DMSO and Potassium Iodide on the Quantitative Precisions (\% CV) of Five HIV Protease Inhibitors ${ }^{a}$

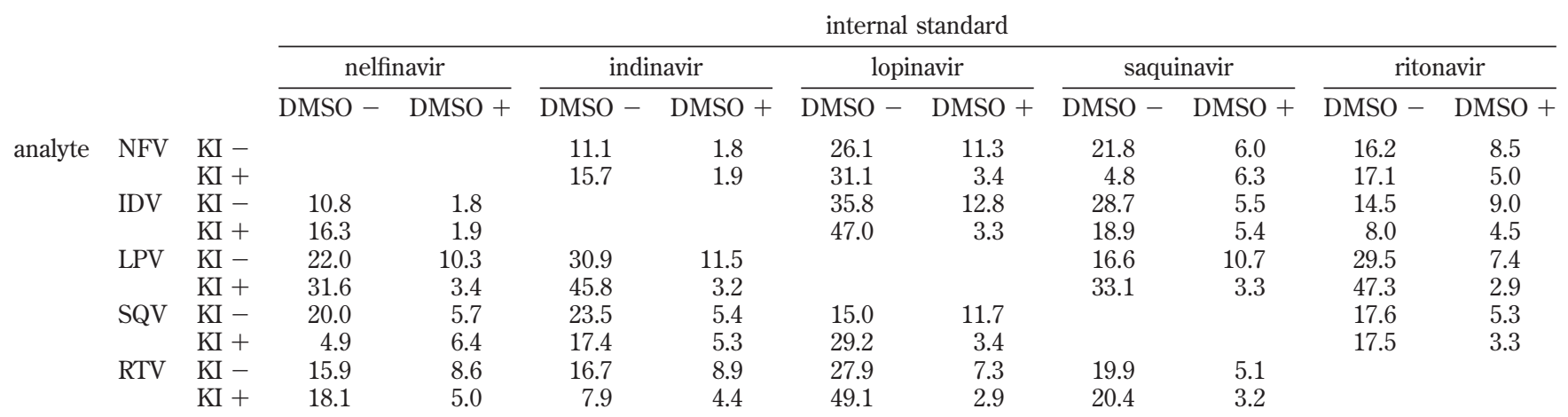

${ }^{a}$ A mixture of five pure HIV-1 protease inhibitors was analyzed using four different DHB preparations (see Experimental Section). One protease inhibitor was chosen as the internal standard, and the remaining four protease inhibitors served as analytes. Subsequently, the analyte-to-internal standard ratios were calculated. The signal-to-noise ratios $(\mathrm{S} / \mathrm{N})$ for the potassiated monoisotopic peaks were used when DHB was used with the addition of potassium iodide. The $\mathrm{S} / \mathrm{N}$ for the protonated monoisotopic peaks were used when DHB was used without the addition of potassium iodide. This procedure was repeated until each protease inhibitor had served as the internal standard. Reported values in the tables are the \% relative standard deviations (RSD) for the analyte-to-internal standard ratios. The concentration per spot on the target plate was 1 pmol for nelfinavir, indinavir, lopinavir, and ritonavir and $250 \mathrm{fmol}$ for saquinavir. Four technical replicates were measured of each sample.

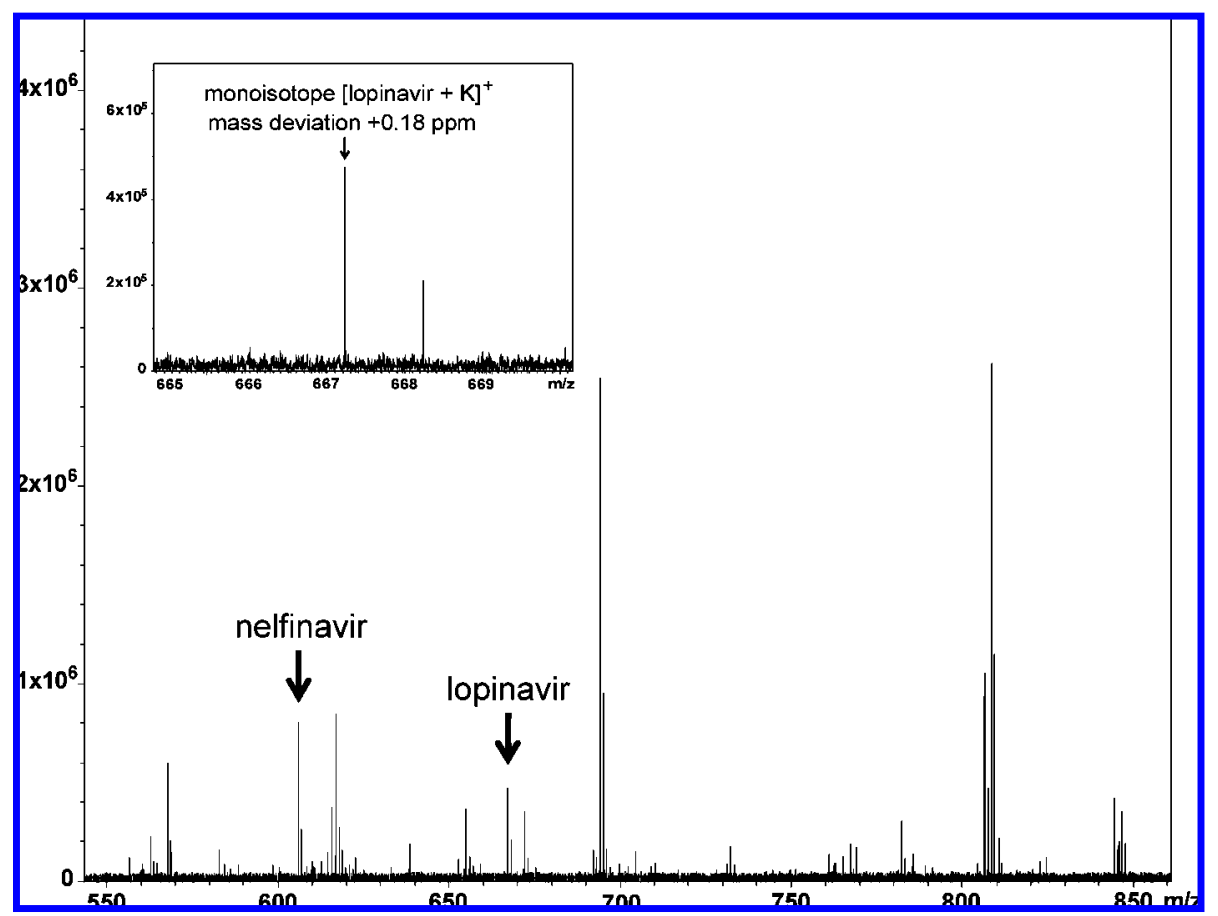

Figure 1. Analysis of lopinavir in a lysate of PBMC. Lopinavir and nelfinavir were spiked in a lysate of $1 \times 10^{6}$ PBMC. The monoisotopic peak of potassiated lopinavir had a S/N of 66 (125 fmol per spot on the target plate). The monoisotopic peak of nelfinavir had a S/N 119 (1 pmol per spot on the target plate). The inset shows a magnification of the lopinavir signal. The measured mass of the monoisotopic peak of potassiated lopinavir was $667.32575 \mathrm{Da}$. The theoretical mass is $667.32563 \mathrm{Da}$ corresponding to a mass deviation of $+0.18 \mathrm{ppm}$. An external mass calibration was used. The $y$-axis is in arbitrary units.

standard) in a lysate of $1 \times 10^{6}$ PBMC (125 fmol lopinavir and 1 pmol nelfinavir per spot on the target plate). It can be seen from this figure that the ionization efficiency of lopinavir is approximately a factor of 4 larger than that for nelfinavir. This factor remains constant over the entire concentration range, and accurate and precise quantitative analysis of the HIV protease inhibitors is thus possible using a chemical analogue as the internal standard.

Table 3 shows the quantitative performance of the MALDIFTICR assay for the five HIV-1 protease inhibitors. The mean quantitative precisions, expressed as \% relative standard deviation, were $6.4 \%$ for saquinavir, $7.3 \%$ for lopinavir, $8.5 \%$ for ritonavir, $11.1 \%$ for indinavir, and 7.2\% for nelfinavir. The mean analyte-to-internal standard ratios of the analysis of three technical replicates were used to calculate the drug concentrations in the samples. The mean quantitative accuracies for the quality control (QC) samples, expressed as \% deviation from the theoretical concentration, were $4.5 \%$ for saquinavir, $6.0 \%$ for lopinavir, $5.9 \%$ for ritonavir, $6.6 \%$ for indinavir, and $8.0 \%$ for nelfinavir. The measured drug concentrations for the individual quality control samples were all within $85-117 \%$ of the theoretical concentrations. The above shows that MALDI-FTICR can be used for accurate quantitative analysis of HIV-1 protease inhibitors in cell lysates. 
Table 3. The Performance of MALDI-FTICR Mass Spectrometry to Quantify Five HIV-1 Protease Inhibitors in PBMC Lysates $^{a}$

\begin{tabular}{|c|c|c|c|c|c|}
\hline compound & $\begin{array}{l}\text { LLOQ } \\
\text { (fmol) }\end{array}$ & $\begin{array}{l}\text { ULOQ } \\
\text { (fmol) }\end{array}$ & $\begin{array}{c}\text { quantitative } \\
\text { precision \% RSD }\end{array}$ & $\begin{array}{c}\text { quantitative } \\
\text { accuracy \% dev }\end{array}$ & $\begin{array}{l}\text { mass deviation } \\
\text { (ppm) }\end{array}$ \\
\hline saquinavir & 4 & 4096 & $6.4(5.1)$ & $4.5(3.0)$ & $-0.05(0.26)$ \\
\hline lopinavir & 16 & 2000 & $7.3(4.2)$ & $6.0(5.2)$ & $+0.11(0.30)$ \\
\hline ritonavir & 31 & 2000 & $8.5(3.8)$ & $5.9(3.6)$ & $-0.21(0.46)$ \\
\hline indinavir & 100 & 6400 & $11.1(4.7)$ & $6.6(4.2)$ & $+0.07(0.14)$ \\
\hline nelfinavir & 100 & 6400 & $7.2(5.1)$ & $8.0(4.0)$ & $-0.28(0.19)$ \\
\hline
\end{tabular}

${ }^{a}$ LLOQ $=$ lower limit of quantification. ULOQ $=$ upper limit of quantification. fmol $=$ femtomole. $\%$ RSD $=$ relative standard deviation of the mean analyte-to-IS ratio for each calibrator in $\% . \% \mathrm{dev}=$ mean absolute deviation of the measured concentration from the real concentration in \%. $\mathrm{ppm}=$ mean deviation of the measured mass from the real mass in parts-per-million. The precisions and accuracies are based on three measurements of each calibrator of each sample set (total of two sample sets). The reported mass deviations are the mean mass accuracies for all measured samples of sample sets 1 and 2 combined. The mean standard deviations are reported between parentheses.

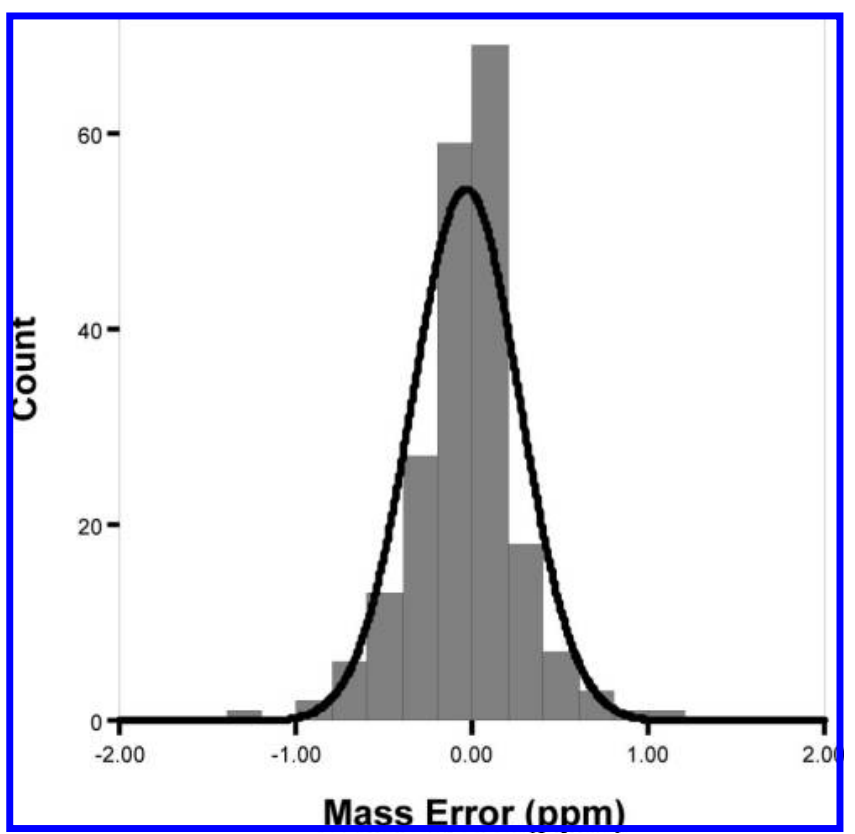

Figure 2. Histogram of mass errors. The histogram shows the mass errors in ppm for the five protease inhibitors (total of 208 measurements) using an external mass calibration. A quadratic mass calibration equation was used. The line shows the Gaussian distribution of the data.

Selectivity. FTICR mass spectrometry provides currently the highest mass accuracy and resolution among mass analyzers. Higher mass accuracy and resolution result in a better selectivity of the assay. We typically obtain resolutions of 100000 (fwhm). The mean mass errors for the HIV-1 protease inhibitors in the QC samples were $-0.05 \mathrm{ppm}$ for saquinavir, $0.11 \mathrm{ppm}$ for lopinavir, $-0.21 \mathrm{ppm}$ for ritonavir, $0.07 \mathrm{ppm}$ for indinavir, and $-0.28 \mathrm{ppm}$ for nelfinavir. Figure 2 shows the histogram of all mass errors for the HIV protease inhibitors in the $\mathrm{QC}$ samples. The average mass error for all measurements was $-0.05 \mathrm{ppm}$, the standard deviation was $0.34 \mathrm{ppm}$, and the root-mean-square was $0.34 \mathrm{ppm}$. These mass errors were obtained using an external mass calibration. Internal calibration could result in even smaller mass errors.

\section{CONCLUSIONS}

We have shown that MALDI-FTICR can be used for precise and accurate quantitative analysis of HIV-1 protease inhibitors in PBMC lysates. This assay was developed for future studies on the intracellular pharmacokinetics of HIV protease inhibitors in
PBMCs obtained from HIV-infected adults and children receiving therapy. The lower limits of quantification needed for such studies are thus a direct consequence of the amount of material that can be obtained from these patients. One million PBMCs can be obtained from a blood sample of $1-2 \mathrm{~mL}$, which is a suitable volume of blood to draw from HIV-1 infected adults and children. The lower limits of quantification (LLOQ) for HIV-1 protease inhibitors in PBMC lysates using the MALDI-FTICR assay were $4 \mathrm{fmol}$ for saquinavir, $16 \mathrm{fmol}$ for lopinavir, $31 \mathrm{fmol}$ for ritonavir, and $100 \mathrm{fmol}$ for indinavir and nelfinavir. These are however the "technical" LLOQs, i.e., the amount of drug in a single spot on the target plate. We dissolved the dried lysates of $1 \times 10^{6} \mathrm{PBMCs}$ in $10 \mu \mathrm{L}$ of solvent and subsequenlty deposited $1 \mu \mathrm{L}$ of this solution on different spots on the target plate. The minimum amount of drug present in the dried lysates should thus be a factor of 10 higher in order for this assay to quantify the drugs. The "biological" LLOQs are thus $40 \mathrm{fmol}$ for saquinavir, $160 \mathrm{fmol}$ for lopinavir, $310 \mathrm{fmol}$ for ritonavir, and $1 \mathrm{pmol}$ for indinavir and nelfinavir per million PBMCs. The reported minimum intracellular concentrations $\left(C_{\min }\right.$ or $\left.C_{\text {predose }}\right)$ of HIV-1 protease inhibitors in HIV- 1 infected adults are $6.4 \mathrm{pmol} /\left(1 \times 10^{6} \mathrm{PBMC}\right)$ for lopinavir, ${ }^{37}$ $850 \mathrm{fmol} /\left(1 \times 10^{6} \mathrm{PBMC}\right)$ for ritonavir, ${ }^{37} 450 \mathrm{fmol} /\left(1 \times 10^{6}\right.$ PBMC) for saquinavir, ${ }^{38} 87 \mathrm{fmol} /\left(1 \times 10^{6} \mathrm{PBMC}\right)$ for indinavir, ${ }^{39}$ and $2.1 \mathrm{pmol} /\left(1 \times 10^{6} \mathrm{PBMC}\right)$ for nelfinavir. ${ }^{40}$ Except for indinavir, the MALDI-FTICR assay can thus be used for quantitative analysis of HIV-1 protease inhibitors in 1000000 PBMCs.

The LLOQs obtained by our MALDI-FTICR assay are comparable to those obtained using the MS-mode of a MALDI-TOF mass spectrometer. ${ }^{8,9}$ The resolving power and mass accuracy of the MALDI-FTICR assay is superior compared to that of a MALDITOF. Thus, using the MS-mode, the MALDI-FTICR assay is more selective than the MALDI-TOF assay. In addition, we found that MALDI-FTICR has a larger dynamic range than MALDI-TOF. ${ }^{8,41}$ Notari et al. used tandem mass spectrometry (MS/MS) on a

(37) Crommentuyn, K. M.; Mulder, J. W.; Mairuhu, A. T.; van Gorp, E. C.; Meenhorst, P. L.; Huitema, A. D.; Beijnen, J. H. Antiviral Ther. 2004, 9, $779-785$.

(38) Ford, J.; Cornforth, D.; Hoggard, P. G.; Cuthbertson, Z;; Meaden, E. R.; Williams, I.; Johnson, M.; Daniels, E.; Hsyu, P.; Back, D. J.; Khoo, S. H. Antiviral Ther. 2004, 9, 77-84.

(39) Hennessy, M.; Clarke, S.; Spiers, J. P.; Mulcahy, F.; Kelleher, D.; Meadon, E.; Maher, B.; Bergin, C.; Khoo, S.; Tjia, J.; Hoggard, P.; Back, D.; Barry, M. Antiviral Ther. 2003, 8, 191-198.

(40) Hennessy, M.; Clarke, S.; Spiers, J. P.; Kelleher, D.; Mulcahy, F.; Hoggard, P.; Back, D.; Barry, M. Antiviral Ther. 2004, 9, 115-122.

(41) Mass Spectrometry: A Textbook; Gross, J. H., Ed.; Springer: New York, 2004; pp 113-130. 
MALDI-TOF/TOF for quantitative analysis of antiretroviral drugs in human plasma and obtained a LLOQ of $2.5 \mathrm{fmol} / \mu \mathrm{L}$ for lopinavir and ritonavir. ${ }^{19}$ To the best of our knowledge, three LC-MS/ MS methods for quantitative analysis of antiretroviral drugs in PBMC have been described in detail. ${ }^{42-44}$ Jemal et al. obtained a LLOQ of $5 \mathrm{fmol} /\left(1 \times 10^{6} \mathrm{PBMC}\right)$ for atazanavir. ${ }^{43}$ Rouzes et al. obtained a LLOQ of $3 \mathrm{pmol} /\left(3 \times 10^{6} \mathrm{PBMC}\right)$ for lopinavir, $1 \mathrm{pmol} /$ $\left(3 \times 10^{6} \mathrm{PBMC}\right)$ for ritonavir, and $2 \mathrm{pmol} /\left(3 \times 10^{6} \mathrm{PBMC}\right)$ for saquinavir. ${ }^{44}$ Colombo et al. obtained LLOQ of $5 \mathrm{fmol}$ for ritonavir, $6 \mathrm{fmol}$ for lopinavir, $6 \mathrm{fmol}$ for saquinavir, $7 \mathrm{fmol}$ for indinavir, and $9 \mathrm{fmol}$ for nelfinavir (minimum amount of quantifiable drug on column). ${ }^{42}$ Our LLOQs obtained for the HIV protease inhibitors in PBMC lysates using MALDI-FTICR are roughly comparable to those obtained by LC-MS/MS. The sample analysis time of the above-described LC-MS/MS methods range from 4 to 20 $\mathrm{min}$. The measurement of one technical replicate takes $3 \mathrm{~min}$ using the MALDI-FTICR assay. In this study, we have measured three technical replicates of each sample, the analysis time for one sample is thus $9 \mathrm{~min}$. This is much slower compared to MALDI-TOF and MALDI-QqQ measurements. ${ }^{1,8}$ Sophisticated spotting devices, such as electrospray sample deposition and inkjet printer technologies, may improve the quantitative precisions of the measurements.

Sample volumes typically used in MALDI mass spectrometry are on the order of $1 \mu \mathrm{L}$. The challenge for successful application of MALDI mass spectrometry for quantitative analysis of drugs

(42) Colombo, S.; Beguin, A.; Telenti, A.; Biollaz, J.; Buclin, T.; Rochat, B.; Decosterd, L. A. J. Chromatogr., B: Anal. Technol. Biomed. Life Sci. 2005, 819, 259-276.

(43) Jemal, M.; Rao, S.; Gatz, M.; Whigan, D. L. Chromatogr., B: Anal. Technol. Biomed. Life Sci. 2003, 795, 273-289.

(44) Rouzes, A.; Berthoin, K.; Xuereb, F.; Djabarouti, S.; Pellegrin, I.; Pellegrin, J. L.; Coupet, A. C.; Augagneur, S.; Budzinski, H.; Saux, M. C.; Breilh, D. L. Chromatogr., B: Anal. Technol. Biomed. Life Sci. 2004, 813, 209-216. is to concentrate analytes in such a small volume in a reproducible manner. In previous studies, we dissolved the dried PBMC lysates in 100 and $25 \mu \mathrm{L}$ and subsequently spotted $1 \mu \mathrm{L}^{8,9}$ In the present study, we dissolved the dried PBMC lysates in $10 \mu \mathrm{L}$ of solvent. We found that dissolving the dried lysates in less than $10 \mu \mathrm{L}$ in a reproducible way is difficult. Spotting larger volumes than $1 \mu \mathrm{L}$ may be used to overcome this problem.

Currently, LC-ESI-MS/MS is the standard for the quantitative analysis of drugs. In this study we have developed a strategy for the quantitative assessment of drugs in biological materials by MALDI-FTICR. Our results demonstrate that MALDI-FTICR mass spectrometry can be successfully used for the precise, accurate, selective, and rapid quantitative analysis of drugs in biological materials. In particular the lower limits obtained for the quantification of HIV-1 protease inhibitors in cell lysates allow clinical application of the MALDI-FTICR technique.

\section{ACKNOWLEDGMENT}

We gratefully acknowledge Aids Fund, The Netherlands (Project 2004051 for J.J.A.v.K. and Project 2005038 for R.A.G.) and Top Institute Pharma (Project T4-212) for their financial support. The consortium of Project T4-212 consists of the Departments of Neurology and Virology of the Erasmus MC (Rotterdam), the Departments of Pediatrics and Clinical Pharmacy of the UMC St. Radboud (Nijmegen), TNO-Quality of Life (Zeist), and GlaxoSmithKline (Zeist) (all from The Netherlands). Antiretroviral drugs were kindly provided by Abbott Laboratories, Merck, Pfizer, and F. Hoffmann-La Roche.

Received for review October 8, 2007. Accepted March 6, 2008.

AC702072C 